STEM, STEAM, STREAM:

Posibilidades, reflexiones y experiencias

\title{
Educación STEAM en educación infantil: Un acercamiento a la ingeniería
}

Recepción: 30/11/2020 | Revisión: 07/02/2021 | Aceptación: 05/08/2021 | Publicación: 01/10/2021

\author{
Ainhoa BERCIANO \\ Universidad del País Vasco/ \\ Euskal Herriko Unibertsitatea \\ ainhoa.berciano@ehu.eus \\ http://orcid.org/0000-0001-7399-4745
}

Clara JIMÉNEZ-GESTAL

Universidad de La Rioja

clara.jimenez@unirioja.es

http://orcid.org/oooo-0003-1766-2855

iD María SALGADo

Universidad de Santiago de Compostela

maria.salgado@usc.es

http://orcid.org/0000-0002-0309-241X

Resumen: En este trabajo planteamos la conveniencia de desarrollar las competencias relacionadas con la Educación STEAM (CTIAM, en castellano) por medio de contextos ricos que favorezcan el aprendizaje. En particular, mostramos cómo el uso de problemas relacionados con la ingeniería, adaptados a las edades de niños y niñas de educación infantil (4 y 5 años), y planteados desde el enfoque de la Educación Matemática Realista, favorece el desarrollo, entre otras cosas, de la competencia matemática, permitiendo trabajar no solo los estándares de contenido sino también los estándares de procesos matemáticos, lo que da lugar a un aprendizaje más significativo.

Palabras clave: STEAM; competencia matemática; estándares de procesos; resolución de problemas; educación infantil.

\section{STEAM EDUCATION IN EARLY CHILDHOOD EDUCATION:} AN APPROACH TO ENGINEERING

Abstract: In this work we propose the convenience of developing the competences related to STEAM Education using rich contexts that favour learning. In particular, we show how the use of problems related to engineering, adapted to the ages of children in Infant Education (4 and 5 years old), and raised from the approach of Realistic Mathematics Education, favours the development, among 
other things, of mathematical competence, allowing to work not only on the content standards but also on the standards of mathematical processes, which results in more significant learning.

Keywords: STEAM; mathematical competence; process standards; problem resolution; early chilhood education.

\section{EDUCACIÓ STEAM A EDUCACIÓ INFANTIL: UN APROPAMENT A L'ENGINYERIA}

Resum: En aquest treball plantegem la conveniència de desenvolupar les competències relacionades amb l'Educació STEAM mitjançant contextos rics que afavoreixin l'aprenentatge. Concretament, mostrem com l'ús de problemes relacionats amb l'enginyeria, adaptats a les edats de nens i nenes d'Educació Infantil (4 i 5 anys), i plantejats des de l'enfocament de l'Educació Matemàtica Realista, afavoreix el desenvolupament, entre altres coses, de la competència matemàtica, permetent treballar els estàndards de contingut $i$ els estàndards de processos matemàtics, cosa que afavoreix un aprenentatge més significatiu.

Paraules clau: STEAM; competència matemàtica; estàndards de processos; resolució de problemes; educació infantil.

\section{Introducción}

En los últimos años está tomando más y más relevancia el concepto de educación STEAM, acrónimo de Science, Technology, Engineering, Art and Mathematics, (CTIAM, ciencia, tecnología, ingeniería, arte y matemáticas en castellano) que pretende integrar estas disciplinas en el proceso educativo y fomentar el desarrollo de cada una de ellas relacionado con el del resto. El planteamiento de experiencias STEAM de calidad en edades tempranas favorece el desarrollo de habilidades en estas materias que facilitarán los aprendizajes posteriores (Zollman, 2012). En educación infantil, y en particular en las disciplinas STEAM, el aprendizaje se produce en situaciones de resolución de problemas, conectadas con la vida cotidiana de niños y niñas (LOE, 2006) y que favorecen la comunicación, lo que facilita, además, el desarrollo de sus habilidades lingüísticas. Según Sarama et al. (2018), todos los niños y niñas tienen la capacidad para aprender y "hacer STEM", porque su curiosidad innata hace que quieran explorar para tratar de entender el mundo que les rodea. En las primeras edades los niños manifiestan interés por elementos del entorno, cuestionan todo, se plantean interrogantes del tipo ¿servirá?, ¿podrá llevar a todos y a todas?, ¿y este botón para qué será? En sus juegos aparecen muchas de las actividades fundamentales en las disciplinas STEAM, como construir, comparar, coleccionar objetos y organizarlos.

Además, si atendemos a la perspectiva de globalidad que nos ofrece el STEAM, si en el diseño de las propuestas matemáticas se incorporan áreas científico-tecnológicas, en especial, la ingeniería y la tecnología, este contexto permitirá fundamentalmente realizar las propuestas por ensayo-error (Alsina, 2020), permitiendo al alumnado tener un papel activo y significativo en el proceso de enseñanza aprendizaje (Couso, 2017), desarrollando estrategias a partir del diálogo, consenso, comprobación, ... para optimizar resultados y desarrollando la competencia de aprender a aprender. Como ejemplo en Educación Infantil, encontramos el trabajo de Alsina y Acosta (2018), en el que el uso del robot educativo ayuda a los niños y niñas a trabajar el reconocimiento de patrones en un contexto novedoso, iniciándose así en el pensamiento algebraico temprano y con respecto a la ingeniería y matemáticas en Alsina (2020) se describe una propuesta de construcción de puentes, desde la observación en el contexto, pasando por el diseño y terminando con la construcción. 
Por otro lado, si añadimos la resolución de problemas matemáticos a este planteamiento, es claro que, por un lado, los niños y niñas aprenderán a tomar decisiones individuales y conjuntas por medio de la resolución (Polya, 1965); y, por otro, el planteamiento de los criterios de selección y resolución deberá atender a varias áreas de conocimiento, claramente diferenciadas y a la vez complementarias. De hecho, al incluir un enfoque más amplio dará lugar a una toma de decisión más globalizada, en la que la comunicación y argumentación juegan un papel fundamental en la discusión grupal entre iguales, ayudando a decir de modo conjunto cuál puede ser la mejor solución desde un planteamiento holístico (Canals, 2010), implicando un aprendizaje integrador de las Ciencias, Tecnología, Ingeniería y Matemáticas (Botero, 2018).

Las investigaciones educativas demuestran que trabajar contenidos matemáticos curriculares en contextos reales permite al alumnado aprender de forma más efectiva y alcanzar un mejor desarrollo académico Alsina (2009). Por este motivo, se considera relevante diseñar e implementar propuestas de matemáticas fundamentadas en los principios de la Educación Matemática Realista (EMR).

Teniendo esto en cuenta, en este trabajo presentamos el diseño teórico, fases de la implementación y los resultados de una actividad diseñada con un enfoque STEAM para el aula de Educación Infantil, y que a su vez está planteada atendiendo los principios de la Educación Matemática Realista. En particular, concluimos que la resolución de problemas relacionados con la ingeniería permite trabajar no solo los estándares de contenido, sino también los estándares de procesos matemáticos, favoreciendo el desarrollo de la competencia matemática.

\section{Marco teórico}

Desde que el National Council for Teachers of Mathematics publicara en el año 2000 sus Principios y Estándares para la Educación Matemática (NCTM, 2000), la comunidad investigadora internacional ha adoptado como válida la idea de que para conseguir una formación adecuada en matemáticas no es suficiente con centrarse en los contenidos, sino que hay que tener en cuenta el modo en que estos contenidos se aprenden y usan. Este documento concreta los estándares curriculares que definen las matemáticas que habría que enseñar, clasificados en estándares de contenido matemático: números y operaciones, álgebra, geometría, medida y análisis de datos y probabilidad; y "las formas de adquisición y uso de estos contenidos" (Alsina y Coronata, 2014), conocidos como estándares de procesos: resolución de problemas, razonamiento y prueba, comunicación, conexiones y representación. Además, según la NCTM (2000), independientemente de la etapa educativa, estos procesos deben capacitar al alumnado a:

\section{Resolución de problemas:}

1. Construir nuevo conocimiento matemático por medio de la resolución de problemas;

2. Resolver problemas que surgen de las matemáticas y en otros contextos;

3. Aplicar y adaptar una variedad de estrategias apropiadas para resolver problemas;

4. Controlar y reflexionar sobre el proceso de resolver problemas matemáticos. 


\section{Razonamiento y demostración:}

1. Reconocer el razonamiento y la prueba como aspectos fundamentales de las matemáticas;

2. Hacer e investigar conjeturas matemáticas;

3. Desarrollar y evaluar argumentos y pruebas;

4. Seleccionar y usar varios tipos de razonamientos y métodos de prueba.

\section{Comunicación:}

1. Organizar y consolidar su pensamiento matemático mediante la comunicación;

2. Comunicar su pensamiento matemático de manera coherente y clara a los compañeros, profesores y otras personas;

3. Analizar y evaluar el pensamiento matemático y las estrategias de los demás;

4. Usar el lenguaje de las matemáticas para expresar ideas matemáticas de forma precisa.

\section{Conexiones:}

1. Reconocer y usar conexiones entre las ideas matemáticas;

2. Comprender cómo se relacionan las ideas matemáticas y se organizan en un todo coherente;

3. Reconocer y aplicar las ideas matemáticas en contextos no matemáticos.

\section{Representación:}

1. Crear y usar representaciones para organizar, registrar, y comunicar ideas matemáticas;

2. Seleccionar, aplicar y traducir representaciones matemáticas para resolver problemas;

3. Usar representaciones para modelizar e interpretar fenómenos físicos, sociales y matemáticos.

En este sentido, numerosas publicaciones recogen la importancia de trabajar los procesos en educación matemática desde los primeros cursos de educación infantil, ya que es en esta primera etapa del desarrollo cuando se sientan las bases sobre las que se construirá el conocimiento matemático. Además, en la declaración conjunta de la National Association for the Education of Young Children y el NCTM (NAEYC y NCTM, 2013) se incide en que son los procesos los que hacen posible la adquisición de los contenidos, así como en la importancia de diseñar situaciones de aprendizaje adecuadas para desarrollarlos.

Centrando nuestro interés en la resolución de problemas, la importancia de esta en educación infantil está ampliamente documentada, tanto en informes de la NAEYC (Copple y Bredekamp, 2009; NAEYC, 2013) o el NTCM (2000), como en investigaciones de expertas y expertos en educación matemática (Bowman et al., 2001; Kilpatrick et al., 2001).

Por otro lado, con el fin de hacer una implementación coherente de la matemática al aula, destacamos el planteamiento de la Educación Matemática Realista (EMR), "la cual se basa en la idea de que la matemática -si ha de tener valor humano- debe estar conectada con la realidad, mantenerse cercana a las niñas y los niños y ser relevante para la sociedad" (Freudenthal, 1977, como se citó en Jiménez-Gestal et al., 2019). Así, la Educación Matemática Realista se basa en seis principios fundamentales (Alsina, 2009): 
- De actividad: Las matemáticas son una actividad humana cuya finalidad es matematizar el mundo que nos rodea.

- De realidad: Las matemáticas se aprenden haciendo matemáticas en contextos reales, entendiendo como reales tanto las situaciones de la vida real del sujeto como aqueIlas que son reales en su mente.

- De niveles: Los alumnos pasan por distintos niveles de comprensión: situacional, en el contexto; referencial, esquematización a través de modelos; general, exploración, reflexión y generalización; y formal, que incluye el conocimiento de los procedimientos estándares y la notación convencional.

- De reinvención guiada: El proceso de aprendizaje permite reconstruir el conocimiento matemático formal.

- De interacción: La interacción entre el alumnado y de éste con el docente provoca la reflexión y propicia que se alcancen mayores niveles de comprensión.

- De interconexión: Los bloques de contenido matemático no pueden ser tratados como entidades separadas.

Desde este enfoque, para el trabajo en las primeras edades es importante considerar en mayor medida los principios de actividad y de realidad, que indican que las matemáticas se aprenden haciendo matemáticas en contextos reales de aprendizaje, así como los principios de interacción e interconexión, que ponen de manifiesto la importancia de las relaciones, tanto entre estudiantes y de estas y estos con la persona docente como de los contenidos entre sí y con aspectos extramatemáticos. Además, cabe destacar como aspecto fundamental de la Educación Matemática Realista el uso de problemas contextualizados en la vida cotidiana para, mediante la interacción en el aula, reinterpretarlos, matematizarlos y desarrollar los conocimientos matemáticos relativos tanto a contenidos como a procesos matemáticos (Freudenthal, 1991).

El trabajo mediante este tipo de actividades requiere de cuatro fases diferenciadas: matematización, trabajo previo en el aula, trabajo en contexto y trabajo posterior en el aula (Alsina, 2011, 2014). La matematización consiste en realizar, por parte de la maestra, un análisis del contexto y descubrir los contenidos y procesos matemáticos que se van a trabajar en la actividad. En esta fase no participan los niños y niñas. El trabajo previo en el aula consiste, esencialmente, en recoger las ideas previas sobre el contexto y los conocimientos matemáticos que vamos a trabajar que tienen las niñas y niños, así como presentar el trabajo en el contexto y planificar las necesidades materiales para el trabajo en el contexto. El trabajo en contexto variará según el contexto elegido, pero en todo caso contemplará descubrir las matemáticas que hay en él y la resolución del problema planteado. En la fase de trabajo posterior en el aula toma relevancia el papel de la comunicación y la argumentación, ya que suele incluir tareas de representación simbólica y explicación de los resultados obtenidos.

Son muchos los trabajos de investigación que muestran las bondades de la implementación de propuestas matemáticas en el aula de Educación Infantil desde el enfoque de la EMR: a nivel de práctica docente (Jiménez-Gestal et al., 2019); desde la perspectiva de la adquisición de los estándares de contenido matemático de modo significativo (Berciano et al., 2016); o desde el tipo de consensos grupales que se pueden dar en situaciones de aprendizaje significativo (Salgado et al., 2020). 
Por otro lado, dentro del enfoque STEAM, son varias las investigaciones que destacan la necesidad de la inclusión de experiencias de ingeniería como recurso metodológico en las implementaciones de las actividades de aula. Esta necesidad ha llevado a la descripción del diseño de ingeniería como un enfoque multifacético que busca que el alumnado desarrolle capacidades para la solución de problemas del mundo real (Cardona et al., 2020). Este se diferencia del método científico en que "el método científico busca responder a una pregunta, mientras que el proceso de diseño en ingeniería busca encontrar soluciones y no cualquier solución, sino la mejor" (Botero, 2018). En este sentido, las implementaciones en el aula basadas en el diseño de ingeniería, en su versión para la etapa educativa infantil, deben estar compuestas por 3 fases: 1) definición del problema, 2) desarrollo de las soluciones y 3) optimización de la solución. Para la etapa educativa infantil, estas fases tienen como objetivo:

- Definición del problema: las niñas y niños deben entender y comprender la naturaleza del problema planteado.

- Desarrollar soluciones: las niñas y niños deben proponer ideas para encontrar la solución. Lo importante es la puesta en común de todos los niños y niñas en grupo aula.

- Optimizar la solución: se debe analizar qué solución es la mejor acorde a unas características predefinidas.

Igualmente, otro aspecto importante en el enfoque STEAM, es la inclusión de la tecnología, que, según Botero (2018), debe garantizar que el alumnado sea capaz de entender las formas en que la tecnología es cada vez más sofisticada y comprender cómo se crea, cómo se adapta a la sociedad y cómo la sociedad la transforma, entre otros.

Aún así, todavía son pocas las publicaciones que abordan la problemática de las propuestas STEAM en el aula de infantil y la adquisición de la competencia matemática en dichas propuestas. Entre ellas, destacamos el trabajo de Alsina y Salgado (2018), en el que ponen de manifiesto como se puede fomentar la competencia matemática en una actividad STEAM, en la que ponen en valor la necesidad de analizar los estándares de procesos matemáticos involucrados en estas propuestas.

\section{Diseño de la experiencia}

En el diseño de la experiencia se tuvieron en cuenta los principios de la EMR, al igual que las fases descritas en el marco teórico. Por otro lado, atendiendo al diseño de ingeniería en STEAM, se vio la necesidad de incorporar las 3 fases de este en el diseño de la actividad, dando lugar a la inserción natural y complementaría siguiente: matematización, trabajo previo en el aula (definición del problema), trabajo en contexto (desarrollo de soluciones) y trabajo posterior (optimizar la solución). Por último, la incorporación de la tecnología en la experiencia se realizó a través del contexto del problema centrado en los robots (descrito en el apartado siguiente). Así, la experiencia de aula se llevó a cabo en un centro público de Galicia, CEIP Sigüeiro, en el aula de 5 años, con un total de 11 niñas y 10 niños. Se implementó a lo largo de 4 sesiones en 4 jornadas distintas. Todas las sesiones se gra-baron en vídeo, se tomaron imágenes $y$, posteriormente, se realizaron las transcripciones de parte de los diálogos por una de las dos docentes que han implementado la experiencia. 


\subsection{Contexto de aprendizaje asociado a la experiencia}

La experiencia diseñada tuvo como contexto de aprendizaje la creación de un robot que pueda llevar a personas (fase 1 del diseño de ingeniería y criterio de inclusión de la tecnología). Dicha creación se realizó en pequeños grupos con unas cajas de construcción STEAM (Figura 1) adecuadas a la edad, y para ella cada grupo debió determinar cuántas personas querían que su robot pudiera llevar y por qué; al igual que las características principales que definen la construcción de su robot (fase 2 del diseño de ingeniería). Finalmente, se pidió que, entre todos los robots construidos, una vez analizadas las características de cada uno, eligieran el que consideran mejor y dijeran el porqué (fase 3 del diseño de ingeniería).

\subsection{Objetivos de la experiencia}

En la Tabla 1 se recogen los objetivos STEAM que se persiguen en cada una de las fases de la experiencia, diferenciando entre los matemáticos, por un lado, y los científicos, tecnológicos y de ingeniería por otro.

\begin{tabular}{|c|c|c|c|}
\hline Fase & Objetivo matemático & $\begin{array}{l}\text { Objetivos científico, } \\
\text { tecnológico, ingeniería }\end{array}$ & $\begin{array}{l}\text { Estructura } \\
\text { docente }\end{array}$ \\
\hline Matematización & $\begin{array}{l}\text { Entender el espacio y las } \\
\text { formas relacionadas con el } \\
\text { mismo en contextos reales y } \\
\text { su medida } \\
\text { Clasificaciones de objetos }\end{array}$ & $\begin{array}{l}\text { Entender conceptos } \\
\text { asociados a la robótica, } \\
\text { física y la ingeniería en } \\
\text { contextos reales }\end{array}$ & $\begin{array}{l}\text { La clase no } \\
\text { participa }\end{array}$ \\
\hline Trabajo previo & $\begin{array}{l}\text { Identificación de ideas } \\
\text { previas sobre características } \\
\text { de clasificación y formas }\end{array}$ & $\begin{array}{l}\text { Identificación de ideas } \\
\text { previas sobre ROBOTS }\end{array}$ & $\begin{array}{l}\text { Gran grupo: } \\
\text { asamblea }\end{array}$ \\
\hline $\begin{array}{l}\text { Trabajo en } \\
\text { contexto }\end{array}$ & $\begin{array}{l}\text { Identificación de objetos } \\
\text { tridimensionales } \\
\text { Clasificación de formas } \\
\text { atendiendo a criterios } \\
\text { matemáticos } \\
\text { Resolución de problemas }\end{array}$ & $\begin{array}{l}\text { Construcción e } \\
\text { identificación de } \\
\text { propiedades fisicas de los } \\
\text { robots y su aplicación } \\
\text { Identificación de aspectos } \\
\text { relacionados con la } \\
\text { ingeniería: eficiencia, } \\
\text { rendimiento, eficacia }\end{array}$ & $\begin{array}{l}\text { Pequeño } \\
\text { grupo }\end{array}$ \\
\hline $\begin{array}{l}\text { Trabajo } \\
\text { posterior }\end{array}$ & $\begin{array}{l}\text { Identificación de criterios } \\
\text { matemáticos } \\
\text { Comunicación, razonamiento } \\
\text { y argumentación matemática }\end{array}$ & $\begin{array}{l}\text { Identificación de criterios } \\
\text { de clasificación STEAM } \\
\text { (robótica, fisica o } \\
\text { ingeniería) } \\
\text { Pensamiento crítico } \\
\text { asociado a rendimiento y } \\
\text { eficacia }\end{array}$ & Gran grupo \\
\hline
\end{tabular}

Tabla 1. Objetivos de aprendizaje en cada fase de la experiencia. 


\subsection{Secuenciación de la experiencia}

La experiencia se diseñó para poder ser llevada a cabo a lo largo de cuatro días, con el fin de establecer conexiones matemáticas $y$, además, poder trabajar otras actividades que fomenten todas las conexiones posibles con otras áreas de conocimiento, no solo las consideradas en el enfoque STEAM, sino también aquellas asociadas a las competencias transversales que deben desarrollar las niñas y los niños (Novo et al., 2017). Así, la distribución de las actividades por día se recoge en la Tabla 2.

\begin{tabular}{|c|c|c|c|c|}
\hline Día & Tiempo/día & Actividad & Tiempo & Estructura \\
\hline 1 & $60 \mathrm{~min}$ & $\begin{array}{l}\text { Acercamiento al concepto de robot } \\
\text { y de su construcción. Juego } \\
\text { exploratorio }\end{array}$ & $60 \mathrm{~min}$ & Pequeño grupo \\
\hline \multirow[t]{4}{*}{2} & \multirow[t]{4}{*}{$100 \mathrm{~min}$} & Clasificación de piezas & $20 \mathrm{~min}$ & Pequeño grupo \\
\hline & & $\begin{array}{l}\text { Toma de decisiones: ¿cuántas } \\
\text { personas llevará nuestro robot? }\end{array}$ & $10 \mathrm{~min}$ & Pequeño grupo \\
\hline & & Creación del robot & $40 \mathrm{~min}$ & Pequeño grupo \\
\hline & & Presentación a la clase & $60 \mathrm{~min}$ & Gran grupo \\
\hline 3 & $60 \mathrm{~min}$ & $\begin{array}{l}\text { Gustómetro ¿cuál me gusta más? } \\
\text { ¿cuál es el mejor? ¿por qué? }\end{array}$ & $60 \mathrm{~min}$ & $\begin{array}{l}\text { Gran grupo } \\
\text { Individual }\end{array}$ \\
\hline 4 & $60 \mathrm{~min}$ & Análisis de las medidas del robot & $60 \mathrm{~min}$ & Individual \\
\hline
\end{tabular}

Tabla 2. Distribución de las actividades por día y sesión.

\subsection{Materiales usados para la experiencia}

Para realizar la experiencia de aula se contó con 4 cajas de construcción STEAM con 116 piezas de diferentes colores y formas. Había cuatro variedades de color: azul, verde, rojo y amarillo; y con respecto a las formas la caja contiene 2 prismas rectangulares, 4 cubos, 36 tiras rectangulares de distintas longitudes y distinto número de orificios, 10 piezas en forma de " $L$ ", 26 tuercas hexagonales, 32 tornillos y 6 ruedas. Además, se utilizaron reglas de medir, hojas para la recogida de los datos y pinturas de colores.

\section{Resultados}

Presentamos a continuación las intervenciones de los niños y niñas y los estándares de procesos que emergen en cada fase de la actividad. Para describir los resultados, en primer lugar, damos una descripción más detallada de la implementación llevada a cabo, poniendo de manifiesto los procesos matemáticos trabajados en cada una de las fases en las que participan.

\subsection{Trabajo previo y procesos matemáticos}

El primer día se dedicó al juego exploratorio, mediante trabajo en pequeño grupo, de 4 o 5 estudiantes, durante 60 minutos.

Se distribuyó el alumnado por el aula y se entregó a cada grupo una caja de piezas de construcción con su libro de instrucciones. Se les invitó a consultar el libro, manipular las piezas con 
la finalidad que descubrieran formas, las compararan, y observaran comportamientos y funcionalidades, entre otros aspectos (Figura 1). El alumnado que quiso, una vez inspeccionado el libreto, construyó lo que consideró.
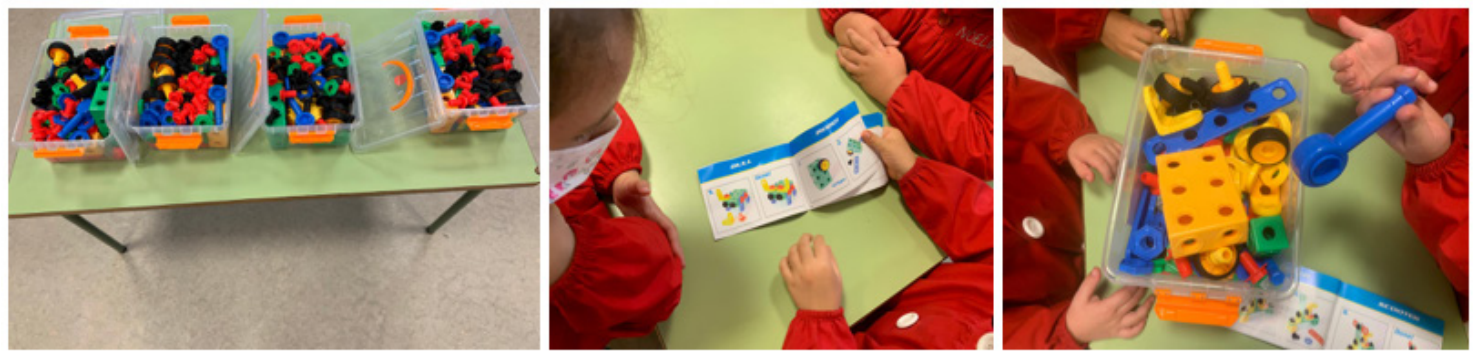

Figura 1. Cajas STEAM, consulta del libro y posterior construcción.

El segundo día se trabajó en pequeño grupo durante 100 minutos distribuidos en 4 actividades, la primera de las cuales forma parte del trabajo previo.

\section{Clasificación, 20 min.}

Se entregó al alumnado la caja de piezas y en una mesa aparte, previa a la construcción del robot, se les invitó a que clasificaran las piezas. No se les dio ninguna instrucción acerca de las características de clasificación, si había de ser por color, forma, tamaño, o cualquier otro criterio, lo que dio lugar a distintas clasificaciones (Figura 2).

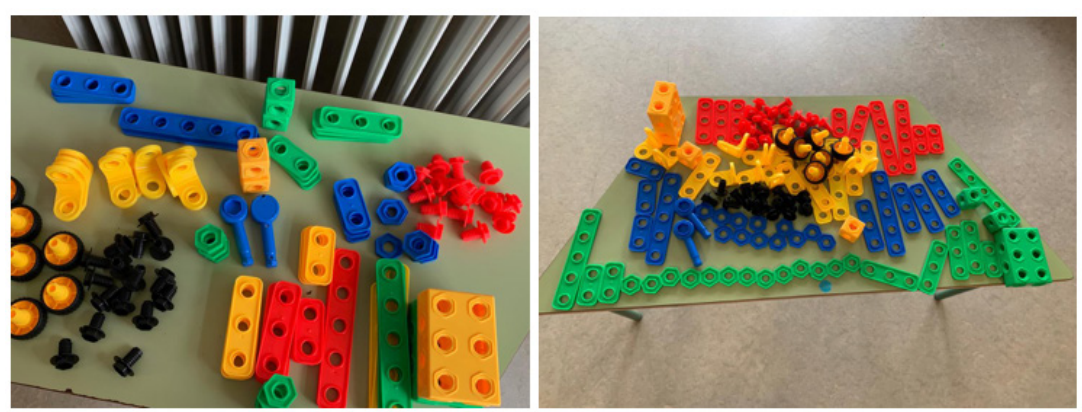

Figura 2. Clasi icación de las piezas de la caja STEAM.

Maestra(M): ¿Qué tenéis para construir vuestro medio para las superthings?

N: Tornillos, ruedas.

M: ¿Y cómo habéis clasificado las cosas, chicos?

N: Por colores.

M: ¿'Solo por colores? Pero mira, aquí hay tornillos rojos y aquí estas piezas, ¿̇por qué las habéis separado si clasificáis por colores? ¿qué más habéis tenido en cuenta? N: Los sillones, cuadrados.

M: ¿Por qué no habéis puesto aquí un tornillo, que es rojo? ¿qué habéis tenido en cuenta? $\mathbf{N}$ : Las piezas así, de color rojo, juntitas. Las que son iguales. 
Este apartado de la actividad principal activa el proceso de razonamiento para realizar la clasificación, además, el diálogo con la maestra sobre la clasificación de las piezas de las cajas STEAM lleva a no solo trabajar contenidos de matemáticas, sino también a tener que verbalizar el criterio utilizado y comprobar si la clasificación realizada se corresponde con el criterio grupal elegido (Figura 3).

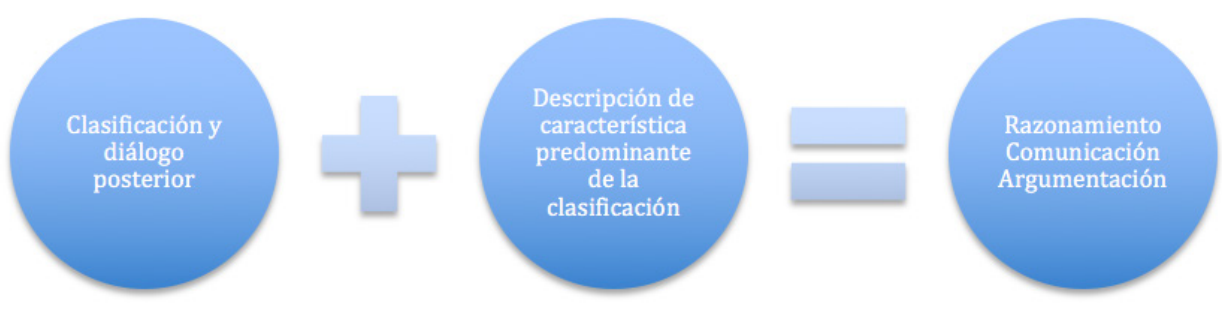

Figura 3. Procesos matemáticos en el trabajo previo.

\subsection{Trabajo en contexto y procesos matemáticos}

El trabajo en contexto comenzó con la toma de decisiones sobre el diseño del robot que debían construir, e incluía la construcción del robot y su presentación al resto de grupos.

Toma de decisiones, 10 min. ¿A cuántas personas quiero que transporte?

Previo a la construcción y diseño de su vehículo, cada equipo tomaba la decisión de a cuántas personas querían que llevara. Esta fase se corresponde con la fase 1 del diseño de ingeniería, en el que se debe entender el problema y establecer condiciones de cumplimiento de la resolución del problema. Cada equipo atendiendo a diferentes criterios determinó el número, resultando en algunos casos 4 (igual que los componentes del equipo) o 9 (porque así eran muchos dentro).

\section{Construcción, $40 \mathrm{~min}$.}

Elaboración en equipo del vehículo para transportar a superthings y designación de nombre y características (Figura 4). En esta parte, el alumnado llevó a cabo la fase 2 del diseño de ingeniería, el desarrollo de las soluciones al problema planteado.
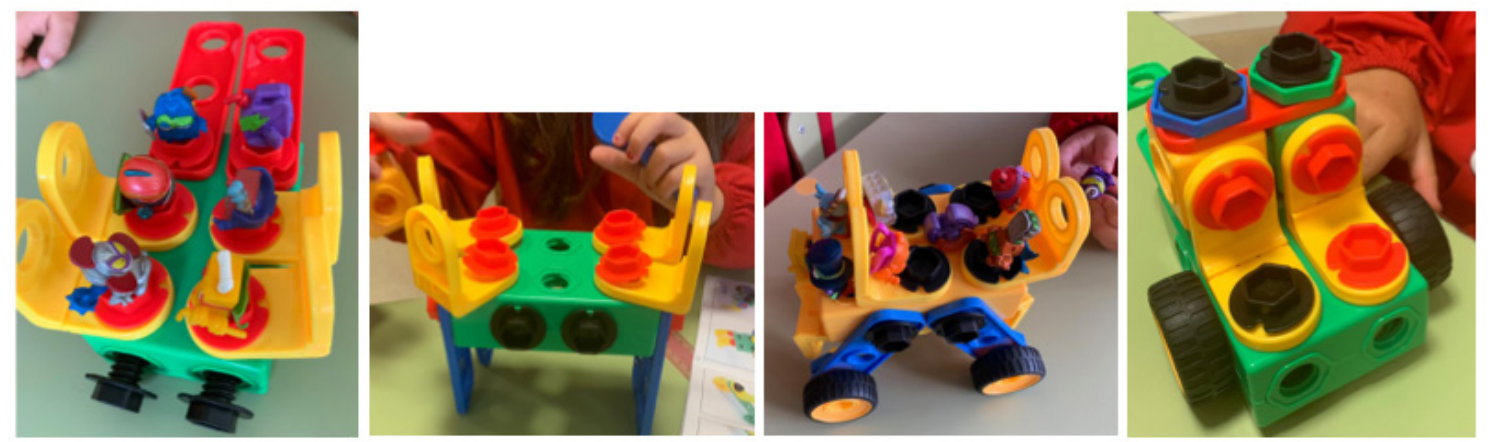

Figura 4. Creación de los robots atendiendo al número de pasajeras y pasajeros. 
Durante la elaboración del robot, la maestra hacía preguntas acerca de la construcción.

Maestra: ¿Cuántos superthings vais a llevar?

N: Nueve.

M: ¿Y cabrán? ¿dónde los vais a llevar?

N: En los sillones.

M: Sí, pero si están boca abajo, ¿̇cómo van? ¿̇e caen?

L: No, pero los ponemos así, como esto aguanta los vamos a poner aquí.

Tras la realización del robot, cada grupo debía explicar las características que tenía a la maestra. En la explicación se debía justificar si el criterio de número de pasajeros elegido se mantiene o no en la creación y por qué.

Maestra: ¿Cómo se llama?

N: Tiburón.

M: ¿Y tiburón por qué?

L: Porque va por el agua, por el fondo donde hay rocas.

M: ¿Y cuántos superthings lleva? Contadme.

N: Nueve.

M: ¿Y tenéis espacio para los nueve?

N: Sí, mira, aquí hay uno, ahí hay dos, hay tres y cuatro y cinco y seis y siete, hay ocho.

M: ¿Y el nueve? ¿dónde va?

N: Aquí.

\section{Presentación en grupo, 30 min. Actividad en gran grupo.}

Cada equipo presentaba al grupo de clase su medio describiendo las características del mismo, como corresponde a la segunda parte de la fase 2 del diseño de ingeniería, la puesta en común de las soluciones. La maestra lo recogió en la pizarra (Figura 5). A medida que se presentaba, la maestra invitaba a reflexionar, comparar y así poder determinar y discriminar beneficios de unos y limitaciones de otros.

Maestra: ¿Qué puede hacer Pinchos?

E: También puede ir por el agua y también puede andar por las nubes y por el aire.

M: ¿Cuántas personas llevaba el vuestro?

E: Cuatro.

M: ¿Cuántas personas lleva tiburón?

E: Nueve.

M: ¿Cuál creéis que es más eficaz?

$\mathbf{N}$ : Tiburón, porque lleva más personas.

M: Mirad, Pinchos caminaba por dos medios, caminaba por el aire y por la tierra, pero ¿cuántas personas lleva pincho?

N: Cuatro.

M: ¿Y tiburón?

N: Nueve.

M: Cuéntame, H, ¿̇qué más cosas hace el vuestro para ser eficiente?

H: Puede ir por la tierra de debajo del agua. 
M: Eso es muy interesante, pero solo se mueve por el agua. ¿Cuántas personas lleva Wisin?

H: Seis.

M: ¿Quién lleva más?

N:Tiburón.

M: ¿¿Qué más cosas hace Wisin?

H: Pues aún no lo hemos pensado, déjanos pensarlo un ratito más.

M: Vale.

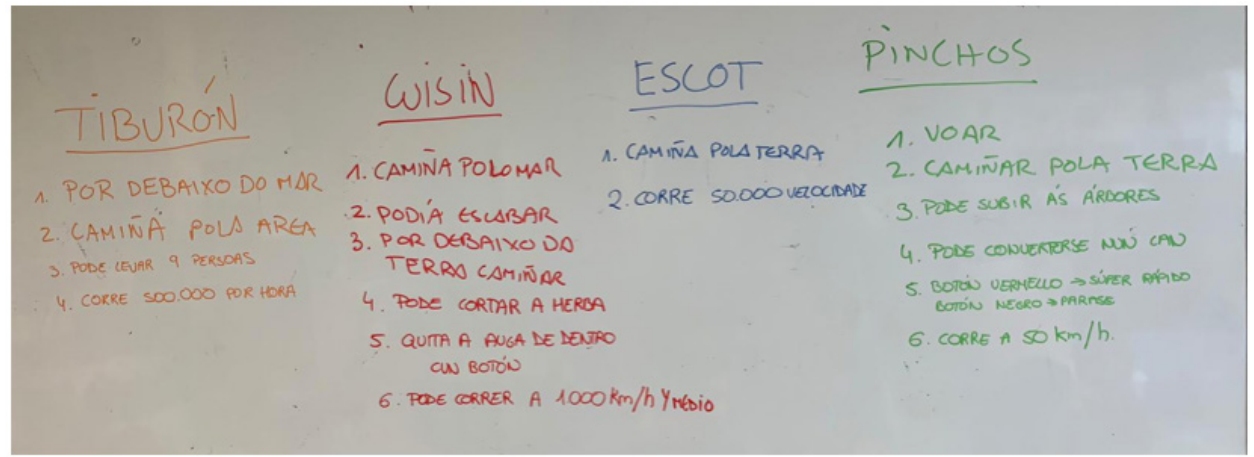

Figura 5. Recopilación de las características en la pizarra.

Esta actividad dio lugar a una descripción de los robots atendiendo a características como ¿cuántas personas puede llevar como máximo?, relacionada con el problema de ingeniería, ¿cómo se mueve? ¿a qué velocidad puede ir?, vinculada a los tipos de movimiento que contempla la física, ¿qué más puede hacer?, asociada a la tecnología, y en la respuesta a esas preguntas se fomentaron los procesos de comunicación y argumentación (Figura 6).

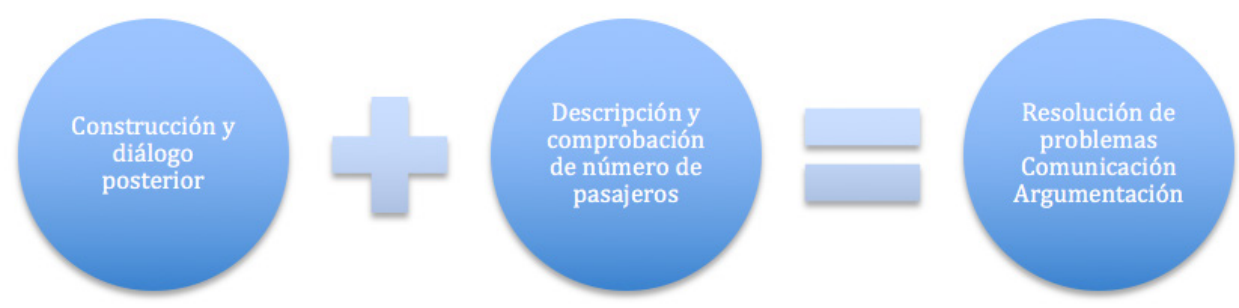

Figura 6. Procesos matemáticos en el trabajo en contexto.

\subsection{Trabajo posterior y procesos matemáticos}

Para el trabajo posterior, se partió del panel elaborado el día anterior en el que se recogían las características de los vehículos creados. 


\section{Gustómetro. ¿Cuál me gusta más? Y ¿cuál es el mejor? 60 min}

Con el fin de llevar a cabo la fase 3 del diseño de ingeniería, la optimización, en gran grupo, la maestra leyó las anotaciones del panel con la intención de recordar las características de los vehículos y tomarlas como punto de partida. A partir de ahí, se invitó a cada niña y niño a que eligiera qué vehículo le gustaba más, exceptuando el suyo propio, se levantara y se pusiera en la fila que corresponda, creando así un diagrama vertical que recogía las preferencias del alumnado.

Para la elección, se invitó a reflexionar si el que más les gusta es el mejor a través de preguntas, ¿por qué te gusta más? ¿qué es lo que hace? ¿por qué te lo comprarías? ¿por qué no este otro?

Maestra: ¿'Tú cuál comprarías?

C: Tiburón, porque lleva muchas personas.

E: Tiburón porque excava.

M: pero tiburón no excava.

E: Pero quiero tiburón.

M: ¿̇por qué?

E: porque nada.

M: Cuéntame.

H: Pincho, porque se convierte en gato

M: ¿y te gustan mucho los gatos? Pero ¿pincho se convierte en gato?

H: No, pero trepa a los árboles y se convierte en perro y me gustan mucho los perros.

M: A ver L., ¿tú cuál prefieres?

L: Escot

M: ¿Por qué Escot?

L:Porque corre mucho

M: ¿Escot corre mucho? Pero ¿̇es el que más corre?

L: No, pero el que más corre es tiburón.

M: Ah, pero vos no podéis votar a tiburón. Vale. Dime N.

N: A pincho, porque si quieres coger castañas él puede subir a los árboles.

M: G. ¿̇cuál crees tú que es el más eficaz?

G: Wizin, porque si quiero buscar un tesoro que está debajo del agua pues lo puedo buscar debajo del agua.

Este diálogo, guiado por parte de la maestra, llevó a que los niños y niñas eligieran una característica predominante del robot, y atendiendo a ella, seleccionarlo, decirlo y justificar su elección, lo que favorece el desarrollo de procesos de argumentación (Figura 7).

Una vez realizada la elección, y con el alumnado colocado en las distintas filas, se provocó la lectura del gustómetro, contando las personas en cada fila y comparando las cantidades para sacar conclusiones sobre la eficacia de cada uno de los diseños, destacando los beneficios de cada uno según la intencionalidad que se quiera. 


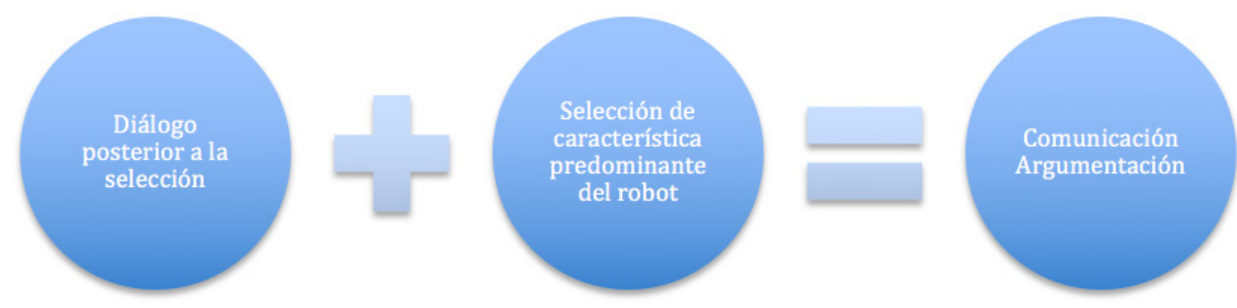

Figura 7: Procesos matemáticos en el gustómetro.

A continuación, se invitó al alumnado a que nuevamente eligiera un vehículo (a excepción del que construyó), pero teniendo en cuenta ahora el que cada cual considere más eficaz, el que se comprarían, indicando previamente el porqué de su elección.
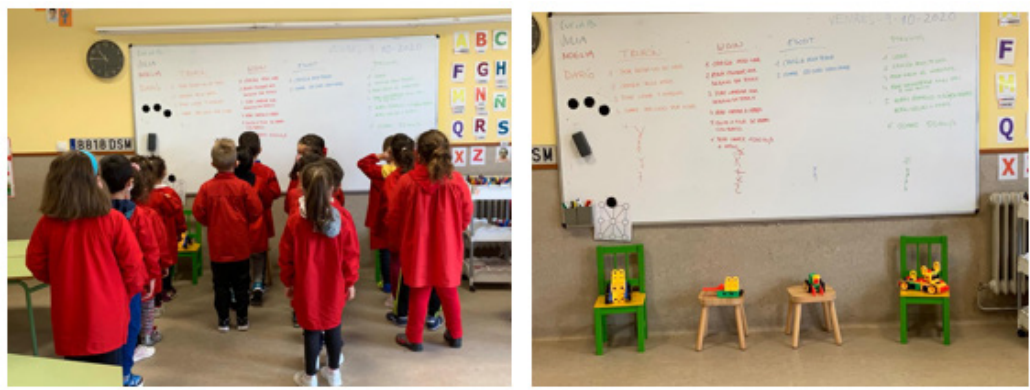

Figura 8. Resultados del gustómetro antes y después del diálogo sobre eficacia.

Como consecuencia del diálogo, algunos de los participantes decidieron cambiar su elección atendiendo a la eficacia percibida, lo que motivó un cambio en los datos asociados a la elección de la clase (Figura 8). Como cierre de la actividad se pidió a cada estudiante que representara los datos en un papel.

\section{Análisis de las medidas del robot $60 \mathrm{~min}$}

Cada estudiante, sentado con su equipo de trabajo, recogió y tomó datos y medidas del vehículo que construyó y lo registró en un papel. Para ello disponían de reglas y cintas métricas, con las cuales pudieron en algún caso añadir datos numéricos a los registros. El objetivo era que el plano y los datos del vehículo permitieran reconstruirlo a posteriori (Figura 9). Como consigna, la maestra indicó que debían realizar algo como el libro de instrucciones que ojearon en un principio. Cuantos más datos ofrecieran y recogieran, más fácil resultaría reconstruirlo y que fuera igual al modelo creado por ellos.
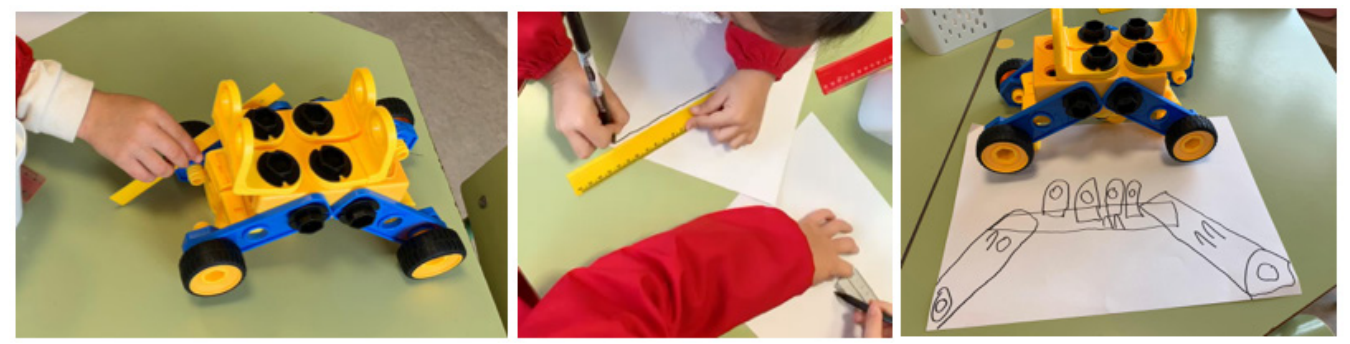

Figura 9. Medidas del robot y su representación gráfica. 
Todo el alumnado utilizó una regla para la toma de medidas, la dificultad surgió en el "cómo", ya que no todos consideraron correctamente el punto de inicio, por lo que no siempre había una correspondencia entre la medida y el valor que registraban. Esto llevó a diferencias en las producciones de los diferentes miembros de los equipos, tanto de la cantidad de datos como del valor.

Por último, de entre todos los planos creados por el equipo, los integrantes del mismo seleccionaron uno, teniendo que llegar a consensos. En un primer momento cada niño creyó que el suyo era el mejor, pero a continuación después de comparar, dialogar y de fijarse en detalles como qué plano tiene "más números", y cuál se semeja más con el real, determinaron el que consideran el mejor. Ayudadas y ayudados con el plano seleccionado, realizaron una presentación en gran grupo al resto de la clase, reflexionando a medida que iban diciendo datos y comparándolos con los de otros equipos.

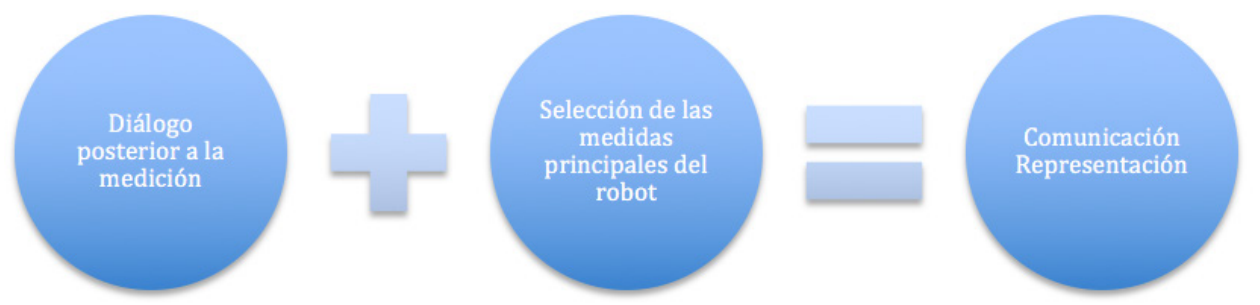

Figura 10. Procesos matemáticos en la medida del robot.

\section{Conclusión}

Como hemos mencionado en apartados anteriores, la enseñanza de la matemática en situaciones contextualizadas es de vital importancia para que el aprendizaje sea significativo (Freudenthal, 1991). Además, gracias a la inclusión del enfoque de aprendizaje STEAM, estos contextos pueden ser enriquecidos con contenido de otras áreas de conocimiento, de modo que el aprendizaje matemático cobre un nivel mayor de significatividad (NAEYC y NCTM, 2013), determinado por fomentar tanto la comunicación como la argumentación desde una perspectiva más holística; donde los criterios de selección en la toma de decisiones vienen condicionados por propiedades de otras áreas, como son la Tecnología, la Ingeniería y la Física.

Así, a lo largo del artículo, hemos puesto de manifiesto cómo una situación cercana, pero a la vez compleja, la construcción de robots y sus características principales, basada en el diseño de ingeniería y la EMR, puede establecer un andamiaje que facilite el aprendizaje de cada una de las áreas STEAM y que, a su vez, las conexiona en un contexto de aprendizaje de Educación Infantil, facilitando el desarrollo y el aprendizaje de todas ellas (acorde con Alsina y Salgado, 2018).

De hecho, debemos mencionar que el objetivo inicial del problema lo lograron todos los equipos, todos elaboraron un medio de transporte para llevar un número de personas determinado (los superthings), a pesar de que el material de construcción era nuevo para los niños y las niñas. Además, las diferencias, las cualidades y las utilidades de cada vehículo ponen de manifiesto las diferencias y preferencias individuales de cada grupo (uno es para nadar, otro resalta que sube a los árboles comparándolo con animales,... ); pero aquí es de vital importancia el uso del lenguaje 
y la interacción en el proceso de enseñanza-aprendizaje, ya que permite que los niños y las niñas reflexionen y sean capaces de llegar a niveles de comprensión más elevados, usando criterios menos egocéntricos y más relacionados con la resolución del problema inicial.

Este hecho se pone claramente de manifiesto cuando se plantea elegir un robot. En este caso, hay que recordar que la actitud crítica de valoración y juicio es una tarea muy difícil en la edad infantil caracterizada por el "yo", por eso cuando al alumnado se le invita a escoger cuál es el mejor, en un primer momento eligen el suyo, por lo que se repite la pregunta por parte de la maestra ampliando la anterior "¿cuál es el mejor excluyendo el vuestro? ¿y por qué?" De este modo, se evita que escojan por afinidades con los compañeros y deban aplicar algún criterio antes consensuado en el grupo clase.

En resumen, la actividad que aquí describimos y analizamos ha llevado a establecer y trabajar las siguientes áreas que se pueden representar como:

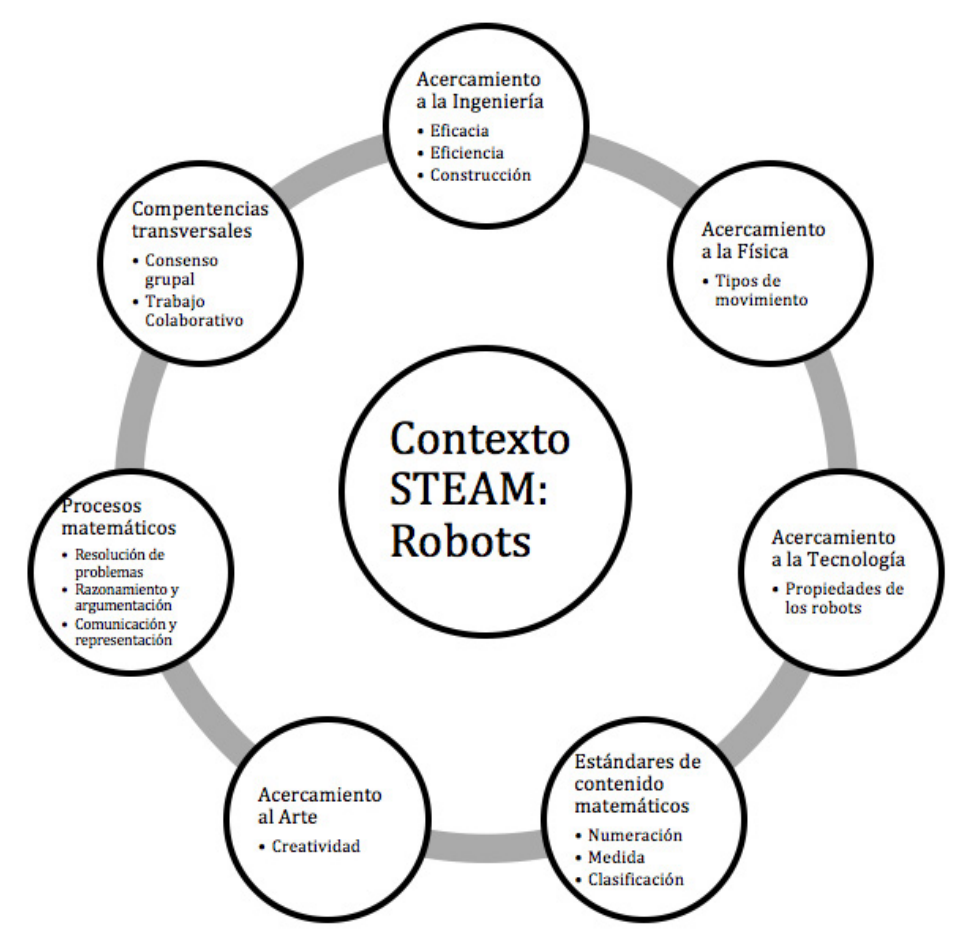

Figura 11. Trabajo en contextos STEAM, contenidos y procesos trabajados.

Por último, no debemos olvidar que esta propuesta es un primer acercamiento al trabajo de la matemática en Educación Infantil desde un enfoque STEAM y, que, por tanto, una mayor investigación de los beneficios y dificultades asociadas a este contexto de aprendizaje se hacen fundamentales en el avance de la enseñanza-aprendizaje de la matemática. 


\section{Referencias}

Alsina, A. (2009). El aprendizaje realista: una contribución de la investigación en Educación Matemática a la formación del profesorado. En M. J. González, M. T. González y J. Murillo (Eds.), Investigación Matemática XIII (pp. 119-127). SEIEM.

Alsina, A. (2011). Educación matemática en contexto: de 3 a 6 años. Cuadernos de Educación.

Alsina, A. (2014). Procesos matemáticos en Educación Infantil: 50 ideas clave. Revista Números, Revista de Didáctica de las Matemáticas, 86, 5-28.

http://www.sinewton.org/numeros/numeros/86/Articulos 01.pdf

Alsina, A. (2020). Conexiones matemáticas a través de actividades STEAM en Educación Infantil. Unión 58, 168-190. https://union.fespm.es/index.php/UNION/article/view/69

Alsina, A. y Acosta, Y. (2018). Iniciación al álgebra en educación infantil a través del pensamiento computacional: una experiencia sobre patrones con robots educativos programables. Unión, 52, 218-236. http://www.fisem.org/www/union/revistas/2018/52/10.pdf

Alsina, A. y Coronata, C. (2014). Los procesos matemáticos en las prácticas docentes: diseño, construcción y validación de un instrumento de evaluación. Edma o-6: Educación Matemática en la Infancia, 3(2), 23-36. http://www.edmao-6.es/index.php/edmao-6/article/view/129

Alsina, A. y Salgado, M. (2018). Land Art Math: una actividad STEAM para fomentar la competencia matemática en Educación Infantil. Edma o-6: Educación Matemática en la Infancia, 7(1),

1-11. http://www.edmao-6.es/index.php/edmao-6/article/view/48

Berciano, A., Jiménez-Gestal, C. y Salgado Somoza, M. (2016). Tratamiento de la orientación en el aula de Educación Infantil desde la perspectiva de la Educación Matemática Realista. Revista Números, 93, 31-44. http://www.sinewton.org/numeros/numeros/93/Articulos_03.pdf

Botero, J. (2018). STEM. Introducción a una nueva forma de enseñar y aprender. STEM Educación Colombia.

Bowman, B. T., Donovan, M. S. y Burns, M. S. (Eds.) (2001). Eager to learn: Educating our preschoolers. National Academy Press.

Canals, M.A. (2010). Problemas y más problemas. Ed. Associació de Mestres Rosa Sensat.

Cardona, M., Arias, V., Trujillo, C. y Carmona-Mesa, J. A. (2020). Divulgación de la ingeniería en estudiantes de secundaria por medio del diseño ingenieril y la educación Maker, una experiencia de campamento bajo el enfoque de educación STEAM. En E. Serna (Ed.), Revolución en la formación y la capacitación para el siglo XXI, Vol. II (pp. 264-277). Editorial Instituto Antioqueño de Investigación. http://doi.org/10.5281/zenodo.4266566

Copple, C. y Bredekamp, S. (2009). Developmentally appropriate practice in early childhood programs serving children birth through age 8 . NAEYC.

Couso, D. (2017). Per a que estem a STEM? Un intent de definer l'alfabetització STEM per a tothom i amb valors. Ciències, 34, 22-30. https://doi.org/10.5565/rev/ciencies.403

Freudenthal, H. (1991). Revisiting mathematics education. China Lectures. Kluwer Academic Publishers.

Jiménez-Gestal, C., Berciano, A. y Salgado, M. (2019). Cómo trabajar la orientación espacial de modo significativo en Educación Inantil: implicaciones didácticas. Revista de Educación Matemática, 31(2),61-74. http://doi.org/10.24844/EM3102.03

Kilpatrick, J., Swafford, J. y Findell, B. (2001. Adding it up: Helping children learn Mathematics. National Academy Press.

LOE (2006). Ley Orgánica 2/2006 de 3 de mayo de Educación. MEC.

National Association for the Education of Young Children and National Council for Teachers of Mathematics (2013). Early childhood mathematics: Promoting good beginnings. A joint position statement. Edma o-6: Educación Matemática en la Infancia, 2(1), 1-23. 
National Council of Teachers of Mathematics. [NCTM] (2000). Principles and standards for school mathematics. Reston: National Council of Teachers of Mathematics (Trad. Castellana, Principios y estándares para la educación matemática. Sociedad Andaluza de Educación Matemática Thales, 2003).

Novo, M.L., Alsina, A., Marbán, J.M. y Berciano, A. (2017). Inteligencia conectiva para la educación matemática infantil. Revista Comunicar, 52, 29-39. https://doi.org/10.3916/C52-2017-03

Polya, G. (1965). Cómo plantear y resolver problemas. Editorial Trillas.

Salgado, M., Jiménez-Gestal, C. y Berciano, A. (2020). Tipos de consensos y estrategias de reparto en pequeños grupos en 4 años: “Operación Lacasitos”. Edma o-6: Educación Matemática en la Infancia, 9(1), 1-11. http://www.edmao-6.es/index.php/edmao-6/article/view/9o

Sarama, J., Clements, D., Nielsen, N., Blanton, M., Romance, N., Hoover, M., Staudt, C., Baroody, A., McWayne, C. y McCulloch, C., (2018). Considerations for STEM education from PreK through grade 3. Education Development Center, Inc. https://www.edc.org/considerations-stem-education-prek-through-grade-3.

Zollman, A. (2012). Learning for STEM literacy: STEM literacy for learning. School Science and Mathematics, 112(1), 12-19. https://doi.org/10.1111/j.1949-8594.2012.00101.X 